\section{P06.06 ADOPTIVE CELL THERAPY OF TRIPLE NEGATIVE BREAST CANCER WITH REDIRECTED CYTOKINE-INDUCED KILLER CELLS}

${ }^{1} \mathrm{R}$ Sommaggio*, ${ }^{2} \mathrm{E}$ Cappuzzello, ${ }^{2} \mathrm{~A}$ Dalla Pietà, ${ }^{2} \mathrm{P}$ Palmerini, ${ }^{2} \mathrm{~A}$ Tosi, ${ }^{1} \mathrm{D}$ Carpanese, ${ }^{3} \mathrm{~L}$ Nicolè, ${ }^{2,4} \mathrm{~A}$ Rosato. 'Veneto Institute of Oncology, IOV - IRCCS, Padova, Italy; ${ }^{2}$ Department of Surgery, Oncology and Gastroenterology, Immunology and Oncology Section, University of Padua, Padova, Italy; ${ }^{3}$ Department of Medicine, Surgical Pathology and Cytopathology Unit, University of Padua, Padova, Italy; ${ }^{4}$ Veneto Institute of Oncology IOV - IRCCS, Padova, Italy

\subsection{6/jitc-2020-ITOC7.85}

Background Cytokine-Induced Killer (CIK) cells share several functional and phenotypical properties of both $\mathrm{T}$ and natural killer (NK) cells, and represent an attractive approach for cell-based immunotherapy as they do not require antigen-specific priming for tumor cell recognition, and can be efficiently and rapidly expanded in vitro. We recently reported that CIK cells have a relevant expression of FcyRIIIa (CD16a), which can be exploited in combination with clinical-grade monoclonal antibodies (mAbs) to redirect their lytic activity in an antigen-specific manner. Here, we report the assessment and the efficacy of this combined approach against triple negative breast cancer (TNBC), an aggressive tumor that still requires reliable therapeutic options.

Materials and methods Different primitive and metastatic TNBC cancer mouse models were established in NSG mice, either by implanting patient-derived TNBC samples or MDA-MB-231 cells subcutaneously or orthotopically into the mammary fat pad, or by injecting MDA-MB-231 cells intravenously. The combined treatment consisted in the repeated intratumoral or intravenous injection of CIK cells and cetuximab, while the mAb alone or CIK cells plus Rituximab served as control treatments. Tumor growth and metastasis were monitored by bioluminescence or immunohistochemistry, and survival was recorded.

Results CIK cells plus cetuximab significantly restrained primitive tumor growth in mice, either implanted with TNBC patient-derived tumor xenografts or injected with MDA-MB231 TNBC cell line. Moreover, in both experimental and spontaneous metastatic models the combined approach almost completely abolished metastasis spreading and dramatically improved survival. The antigen-specific mAb favored tumor and metastasis tissue infiltration by CIK cells, and in particular led to an enrichment of the CD16a ${ }^{+}$subset.

Conclusions Data highlight the potentiality of a novel immunotherapy approach where a non-specific cytotoxic cell population can be converted into tumor-specific effectors with clinicalgrade antibodies, thus providing not only a therapeutic option for TNBC but also a valid alternative to more complex approaches based on chimeric antigen receptor-engineered cells. Disclosure Information R. Sommaggio: None. E. Cappuzzello: None. A. Dalla Pietà: None. P. Palmerini: None. A. Tosi: None. D. Carpanese: None. L. Nicolè: None. A. Rosato: None.

\section{P06.07 CXCR6 EXPRESSION ENHANCES ACCUMULATION OF ANTI-MESOTHELIN CAR T CELLS AT THE TUMOR SITE AND THEIR THERAPEUTIC EFFICACY IN PANCREATIC CANCER XENOGRAFTS}

'S Stoiber, 'S Lesch, '1 Ogonek, 'B Cadilha, 'M Benmebarek, 'A Gottschlich* ${ }^{1} \mathrm{P}$ Metzger, ${ }^{1} \mathrm{C}$ Hörth, ${ }^{1} \mathrm{~A}$ Nottebrock, ${ }^{1,2} \mathrm{~S}$ Endres, ${ }^{1,2} \mathrm{~S}$ Kobold. ${ }^{1}$ Center of Integrated Protein Science Munich and Division of Clinical Pharmacology, Department of Medicine IV, Klinikum der Universität München, Member of the German Center for Lung Research, Munich, Germany; ${ }^{2}$ German Center for Translational Cancer Research (DKTK), Munich, Germany

10.1136/jitc-2020-ITOC7.86
Background Chimeric antigen receptor (CAR) T cell therapy is currently approved for the treatment of some hematological malignancies. However, CAR T cells have so far lacked efficacy in the treatment of solid tumors. A major hurdle of CAR T cell therapy is the limited infiltration of CAR $\mathrm{T}$ cells into tumor tissue. Chemokine receptors enable immune cells to migrate along a chemokine gradient. Here, we show that overexpression of the C-X-C chemokine receptor 6 (CXCR6) enhances CAR $\mathrm{T}$ cell accumulation in $\mathrm{C}-\mathrm{X}-\mathrm{C}$ motif ligand 16 (CXCL16)-positive xenograft pancreatic cancer models, resulting in increased anti-tumor potency of anti-mesothelin CAR T cells. Materials and Methods Human T cells were retrovirally transduced with an anti-mesothelin CAR and CXCR6. NSG mice were injected subcutaneously with mesothelin-CXCL16-overexpressing tumor cells. Mice were treated once with CAR-, CAR-CXCR6- or mock-transduced T cells when tumors were palpable and tumor size was monitored with a caliper. In a separate tracking experiment, subcutaneous tumors were established as described above and the presence of $\mathrm{T}$ cells at the tumor site was determined by FACS analysis within one week after adoptive $\mathrm{T}$ cell transfer. For orthotopic xenograft experiments mesothelin-CXCL16-overexpressing tumor cells were directly injected into the pancreas of NSG mice and one-time treatment with CAR-, CAR-CXCR6- or mock T cells was performed 5 days post tumor injection.

Results In a subcutaneous xenograft model of pancreatic cancer CXCR6-expressing CAR T cells displayed improved anti-tumoral potency compared to CAR T cells without CXCR6, resulting in prolonged survival of mice and tumor clearance in 9 out of 10 CAR-CXCR6-treated mice. A tracking experiment confirmed the increased accumulation of CAR-CXCR6 T cells compared to CAR $\mathrm{T}$ cells at the subcutaneous tumor site, suggesting increased migratory capacity of CAR-CXCR6-transduced T cells towards CXCL16-expressing tumors as the mode of action. Treatment of orthotopic pancreatic cancer xenografts similarly revealed prolonged survival of CAR-CXCR6-treated animals in comparison to CAR-treated animals, suggesting improved antitumor efficacy of CAR-CXCR6-transduced T cells.

Conclusions Forced expression of CXCR6 in anti-mesothelin CAR T cells increased the accumulation of CAR T cells at the CXCL16-positive tumor site, resulting in improved survival of treated mice and in complete tumor rejection in the majority of cases. This data reveals the potential of CXCR6 to direct CAR $\mathrm{T}$ cells to the tumor site and this approach may therefore be an attractive strategy to target a major pitfall in the translation of CAR T cell therapy to solid tumors.

Disclosure Information S. Stoiber: None. S. Lesch: None. J. Ogonek: None. B. Cadilha: None. M. Benmebarek: None. A. Gottschlich: None. P. Metzger: None. C. Hörth: None. A. Nottebrock: None. S. Endres: None. S. Kobold: None.

\section{P06.08 IMMUNOMODULATORY BIOMARKERS IN NEOADJUVANT CHEMOTHERAPY OF BREAST CANCER}

${ }^{1} \mathrm{~K}$ Wimmer ${ }^{*},{ }^{2} \mathrm{M}$ Sachet, ${ }^{1} \mathrm{R}$ Exner, ${ }^{1} \mathrm{~F}$ Fitzal, ${ }^{3} \mathrm{M}$ Filipits, ${ }^{2} \mathrm{R}$ Oehler. ${ }^{1} \mathrm{Medical}$ University Vienna, Department of Surgery and Comprehensive Cancer Center, Vienna, Austria; ${ }^{2}$ Department of Surgery - Research Laboratories and Comprehensive Cancer Center, Anna Spiegel Center of Translational Research, Vienna, Austria; ${ }^{3}$ Medical University Vienna, Institute of Cancer Research, Department of Medicine I, Vienna, Austria

10.1136/jitc-2020-ITOC7.87

Background Neoadjuvant chemotherapy (NAC) with epirubicin/cyclophosphamid followed by docetaxel $(\mathrm{E} / \mathrm{C}->\mathrm{D})$ is 
currently one standard-of-care therapy option in women with early, high-risk or locally advanced breast cancer. While some patients respond excellently to preoperative therapy, in other patients significant tumor shrinkage cannot be achieved. We investigated the impact of NAC on circulating immunomodulatory parameters. We also examined whether changes in these parameters correlate with the response to NAC measured by the Residual Cancer Burden (RCB) score determined after neoadjuvant treatment.

Materials and Methods To detect drug-specific effects, two different NAC regimens in primary breast cancer patients scheduled to pre-operative therapy were compared. 39 patients with conventional anthracycline/taxane sequence $(\mathrm{E} / \mathrm{C}->\mathrm{D}$, $n=39)$ and 40 patients with reverse sequence $(D->E / C)$ were included. Blood plasma samples were collected at three time points - 'baseline' (before NAC), 'midterm' (after the first six cycles of NAC) and 'surgery' (after NAC before operation). The plasma levels of uPA, uPAR, TIM-3, MCP-1, MCP-2, OPG, IP-10, CD 27, Eotaxin, Tweak, TRAIL, PD-L2, M-CSF and VEGF-A were determined either by using ELISA or a multiplex bead array immunoassay.

Results OPG, CD27, MCP-1, MCP-2, CCl19, Tweak, TRAIL, PD-L2 and M-CSF decreased between baseline and midterm in $\mathrm{E} / \mathrm{D}->\mathrm{D}$ patients. However, the majority of patients treated with the reverse sequence showed no such effect. These druginduced changes correlated with the RCB score. Non-responders $(\mathrm{RCB} \geq 1.36)$ showed a significantly different pattern than responders.

Conclusion These data confirm that NAC affects the immune system in a drug-specific manner. Factors correlating with the RCB-score might represent promising biomarkers to predict the response to therapy.

Disclosure Information K. Wimmer: None. M. Sachet: None. R. Exner: None. F. Fitzal: None. M. Filipits: None. R. Oehler: None.

\section{P06.09 ANTI-HPSMA CAR ENGINEERED NK-92 CELLS: AN OFF-THE-SHELF CELLULAR THERAPEUTIC FOR TARGETED ELIMINATION OF PROSTATE CANCER CELLS}

${ }^{1} \mathrm{G}$ Zuccolotto*, ${ }^{2} \mathrm{~A}$ Penna, ${ }^{2} \mathrm{IM}$ Montagner, ${ }^{2} \mathrm{D}$ Carpanese, ${ }^{1} \mathrm{~A}$ Rosato. ${ }^{1}$ Università Degli Studi di Padova, Padova, Italy; ${ }^{2}$ Istituto Oncologico Veneto, Padova, Italy

\subsection{6/jitc-2020-ITOC7.88}

Background Adoptive cell therapy of malignant diseases takes advantages of the cellular immune system to recognize and destroy cancer cells. Despite the remarkable success in B cell malignancies after adoptive transfer of CD19 CAR T cells, CAR $\mathrm{T}$ cell therapy in solid tumors has shown less encouraging clinical results, above all caused by tumor escape mechanisms.In order to overcome such limitations, NK-92, a permanent and IL-2-dependent cell line with a high cytotoxicity in vitro, has been engineered in preclinical models with CAR. In this project, we exploited a CAR directed against the human antigen hPSMA that is overexpressed in prostate tumors. This project aimed at transducing NK-92 cell line to obtain a hPSMA-specific CAR NK-92 cell population, to be thereafter characterized in vitro and in vivo for antigen-specific functional activity.

Materials and Methods NK-92 cell line was transduced with a lentiviral vector (LV) carrying a CAR anti-hPSMA. The cell population obtained was then sorted and analyzed for degranulation capacity, IFN $\gamma$ production and lytic activity against hPSMA $^{+}$(PC3-hPSMA, LNCaP) or hPSMA-tumor cell lines. In vivo therapeutic efficacy of CAR-transduced NK-92 was evaluated initially using Winn-Assay and than in subcutaneous and orthotopic tumor models.

Results CAR-expressing LV efficiently transduced NK-92 cells, which in turn produced cytokines, degranulated and exerted a relevant cytotoxic upon challenge with PSMA+ prostate tumor cells, irrespective of $10 \mathrm{~Gy} \gamma$-irradiation. In all the in vivo, tumor models CAR-transduced NK-92 shown a statistically significant inhibition of tumor growth.

Conclusions Chimeric antigen receptor-engineered NK-92 could offer a valid and cost-effective alternative to primary CAR NK or $\mathrm{T}$ cells, in particular in cases, where a suitable donor is not available or the sophisticated infrastructure needed for cell isolation, expansion and genetic modification is missing. This work demonstrates that CAR-engineered NK92 cells display a high and specific recognition of hPSMA+ PC both in vitro as is in vivo, and could represent an efficient strategy as a new therapeutic intervention against prostate carcinoma, thus paving the way to an Off-The-Shelf cellular therapeutic for targeted elimination of cancer cells and induction of protective antitumor immunity.

Disclosure Information G. Zuccolotto: None. A. Penna: None. I.M. Montagner: None. D. Carpanese: None. A. Rosato: None.

\section{P06.10 SHORT TERM INHIBITION OF CHECKPOINT PROTEINS INCREASES EX VIVO EXPANSION OF TUMOUR INFILTRATING LYMPHOCYTES IN HIGH GRADE SEROUS OVARIAN CANCER}

${ }^{1} \mathrm{CA}$ Waddell*, ${ }^{1} \mathrm{MJ}$ Price, ${ }^{1} \mathrm{P}$ Johnson, ${ }^{1,2} \mathrm{RJ}$ Edmondson, ${ }^{1,2} \mathrm{GL}$ Owens. ${ }^{1}$ University of Manchester, Manchester, UK; '2Saint Mary's Hospital, Manchester, UK

\subsection{6/jitc-2020-ITOC7.89}

Background Ovarian cancer is the most lethal gynaecological malignancy, accounting for approximately 185,000 deaths worldwide in 2018. The majority of patients will experience recurrence of disease. Therefore, there is an urgent need for the development of further therapies to improve patient survival. Tumour infiltrating lymphocyte (TIL) therapy has shown clear efficacy in immunogenic cancers, and TIL can be readily expanded ex vivo from samples of high grade serous ovarian cancer (HGSOC). Key indicators of effective TIL products for infusion are high TIL yield and functionality against autologous tumour. Blockade of checkpoint proteins is effective in increasing TIL yield and functional response from ovarian cancer TIL cultures. However, it is unknown whether blockade of other key checkpoints, including programmed death ligand1 (PD-1), T cell immunoglobulin mucin-3 (TIM-3) and lymphocyte activation gene-3 (LAG-3) increase TIL yield in ex vivo cultures from HGSOC samples.

Materials and Methods TIL cultures were generated from surgically resected HGSOC tumour samples and were incubated with CD3/CD28 Dynabeads. 3000IU/mL recombinant interleukin-2 (IL-2) was added on alternate days for 7 days before beads were removed. $1000 \mathrm{IU} / \mathrm{mL}$ IL-2 was added on alternate days for a further 12 days of culture. In cohort $1,10 \mu \mathrm{g} / \mathrm{mL}$ $\alpha$ PD-1, $\alpha$ TIM-3 or $\alpha$ LAG-3 antibodies were added at initiation of TIL cultures only. In cohort $2,10 \mu \mathrm{g} / \mathrm{mL} \alpha \mathrm{PD}-1$, $\alpha$ TIM-3 or $\alpha$ LAG-3 antibodies were added on alternate days 